\title{
Automated Crystallography and Grain Mapping in the TEM
}

\author{
F. Bo Clayton ${ }^{1}$, Brian W. Kempshall ${ }^{2}$, Stephen M. Schwarz ${ }^{2}$, and Lucille A. Giannuzzi ${ }^{2}$ \\ ${ }^{1}$ Lake Highland Preparatory School, Orlando, FL \\ ${ }^{2}$ Mechanical Materials and Aerospace Engineering, University of Central Florida, Orlando, FL \\ $32816-2450$
}

Obtaining crystallographic information automatically has become routine using computer acquisition of electron backscatter diffraction (EBSD) patterns in the scanning electron microscope (SEM) [1]. The crystallographic information that is acquired may be used to generate data from polycrystalline samples that include crystallographic texture information, grain boundary misorientation analysis, phase identification, grain size distribution, and grain mapping. A natural extension of the SEM analysis is to automate the acquisition of crystallographic information obtained from the transmission electron microscope (TEM). This has recently become a reality via a commercially available system. The system automatically acquires dark field images using conical scan methods. The dark field images are then used to reconstruct diffraction patterns for computer indexing. Once an orientation is assigned for each pixel, statistical manipulation of the data may be performed as with the EBSD data. In addition, grain maps may be obtained without any a priori knowledge of the orientation of each pixel. That is, just the position of the brightest reflectors from the reconstructed diffraction pattern may be used to differentiate between grains. Automatic grain mapping via crystallographic methods is a major breakthrough for TEM analysis since accurate grain size determination via the TEM often requires tedious analyses of multiple images obtained at different tilt conditions due to diffraction contrast effects. The following results were obtained using a Philips/TEM Tecnai F30 TEM operating at $200 \mathrm{keV}$ equipped with an EDAX/TSL ACT system.

Two different samples were used for the study, the surface of abalone (Nacre) and a Cu bicrystal consisting of a $45^{\circ} /[100]$ twist boundary [2]. TEM specimens were prepared using an FEI 200TEM focused ion beam workstation equipped with an Omniprobe in-situ W probe. The abalone was prepared using the ex-situ lift-out technique [3]. The $\mathrm{Cu}$ bicrystal was prepared using the in-situ liftout technique [4].

FIG. 1 shows a cross-section TEM bright field (BF) image from the abalone. The abalone consists of layers of $\mathrm{CaCO}_{3}$ (aragonite) separated by organic material [5]. An inset of a [010] selected area diffraction pattern from the dark grain in the Laue condition (and overlapping grains) is shown in FIG. 1. FIG. 2 is a grain map of the abalone using the ACT system. The grain boundaries within the $\mathrm{CaCO}_{3}$ are readily observed as shown by the arrows and the apparent texture of the abalone is evident by the similar colors of contrast that is produced by the grain map. FIG. 3 is a processed

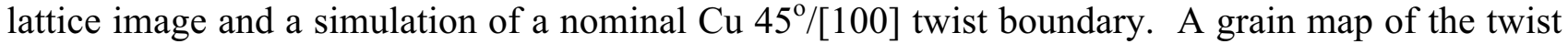
boundary at a lower magnification is shown in FIG. 4. A grain boundary misorientation of $43.9^{\circ} /\left[\begin{array}{lll}1 & 30 & 0\end{array}\right]$ was obtained using reconstructed diffraction patterns. This compares well with a value of $44.7^{\circ} /[100]$ that was obtained previously using the EBSD technique [2]. The acquisition of automatically obtained crystallographic information in the TEM will greatly aid in the microstructural evaluation of TEM specimens [6]. 

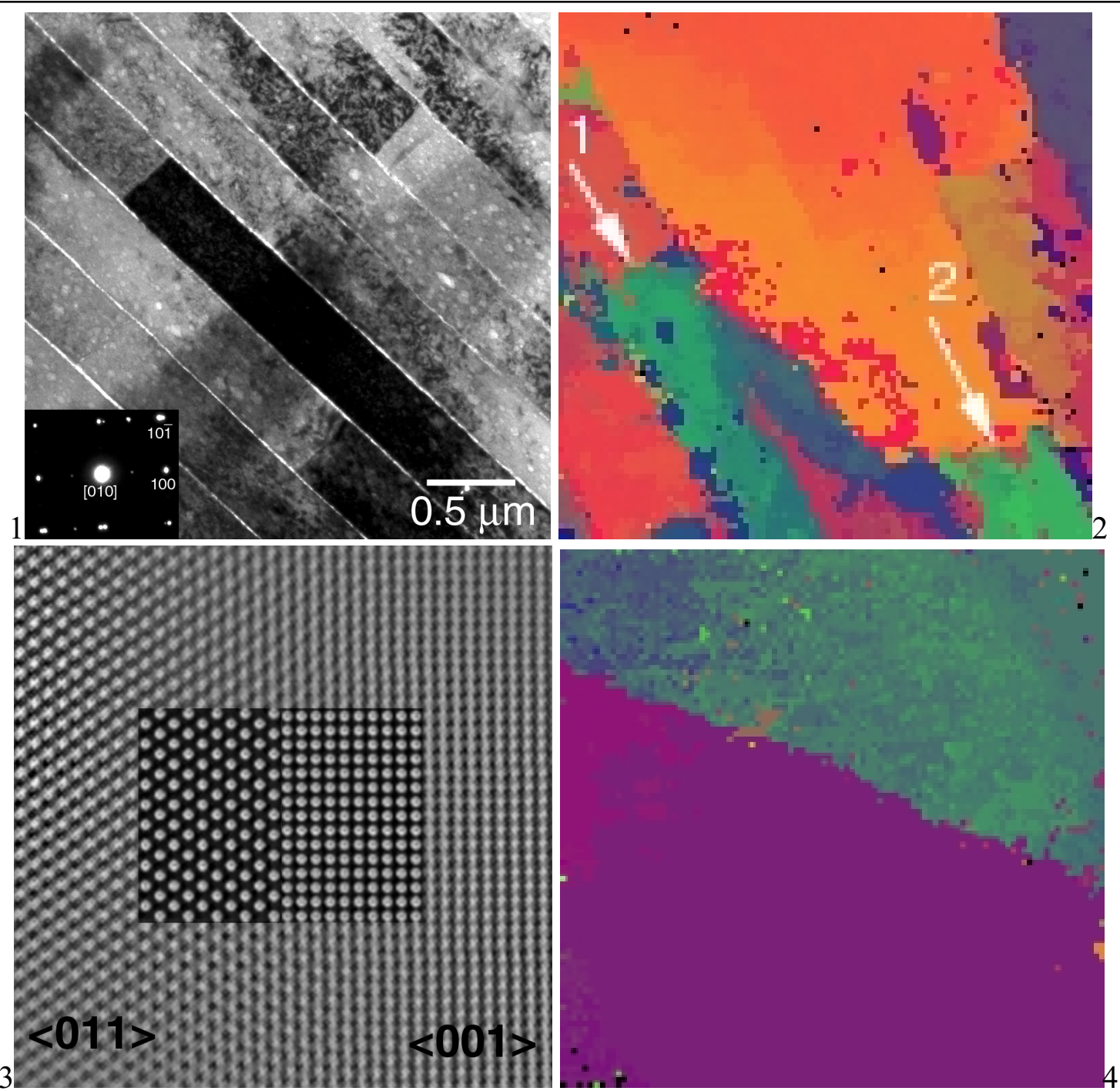

FIG. 1. BF TEM image of abalone.

FIG. 2. A grain map of abalone.

FIG. 3. BF TEM image and HREM image of a $\mathrm{Cu} 45^{\circ} /[100]$ twist boundary.

FIG. 4. A grain map of the $\mathrm{Cu}$ bicrystal.

\section{References}

[1] A.J. Schwartz et al., EBSD in Materials Science, (2000), Kluwer/Plenum, New York.

[2] S.M. Schwarz et al., J. Crystal Growth, 222 (2001) 392.

[3] L.A. Giannuzzi, et al., Mat. Res. Soc. Symp. Proc., 480 (1997) 19.

[4] T. Kamino et al., Microsc. Microanal., 6 (Suppl. 2 ) (2000) 510.

[5] N. Yao et al., Microsc. Microanaly. 6 (Suppl. 2) (2000) 896.

[6] The support of NSF DMR \#9703281 and Omniprobe is gratefully appreciated. 